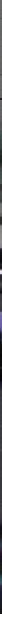

\title{
Der Nationale Krebsplan trägt Früchte
}

Mit dem dritten großen Anlauf - die regionalen Bemühungen gar nicht eingerechnet - scheint die deutsche Onkologie endlich international mündig zu werden: Nach frustranen Versuchen 1979 (Arbeitsgemeinschaft Deutscher Tumorzentren ADT) und 1990 (Übernahme der DDR-Register) hat das Bundeskabinett im Einvernehmen mit der Bundesgesundheitsministerkonferenz und dem Bundesrat beschlossen, das im Nationalen Krebsplan (NKP) 2009 in Ziel 8 geforderte flächendeckende Klinische Krebsregister (KKR) einzuführen. Im Rahmen des Krebsplan-Umsetzungsgesetzes wird nun unter anderem der Aufbau regionaler, bevölkerungsbezogener klinischer Krebsregister mit einheitlichem Datensatz verpflichtend werden.

In einigen Bundesländern bestehen epidemiologische Krebsregister teilweise schon seit einem Dreivierteljahrhundert, überwiegend von den Landesregierungen unterhalten. Doch der Widerstand gegen den seit über 30 Jahren von der Deutschen Krebsgesellschaft (DKG) geforderten Ausbau zu klinischen Registern wurde zu einer typisch deutsch-föderalen Posse, an der verschiedene Interessengruppen beteiligt sind:

- Der Datenschutz, dem der Schutz der Daten wichtiger scheint, als der von Menschenleben.

- Die Krankenkassen, die zwar den Nutzen erkannten, aber die vergleichsweise geringen Kosten anderen anlasten wollten.

- Die KVen, die die Verpflichtung zur Dokumentation wieder aus der Onkologie-Vereinbarung löschten, die 1982 von der Arbeitsgemeinschaft Internistische Onkologie (AIO) der DKG mit der Kassenärztlichen Bundesvereinigung (KBV) und der Gesetzlichen Krankenversicherung (GKV) eingeführt wurde, und den Dokumentationsaufwand vorschieben.

- Schließlich die Landesregierungen, die teils aus parteipolitischen, teils auch aus Kostengründen die vielfältigen Initiativen abwiesen.

Und schon wieder tönt der AOK-Bundesverband, dass „dadurch für die Krebspatienten nichts besser“ werde - trauriger Vorwand, die Finanzierung (von geplant pauschal 94 Euro pro neuem Fall) in Frage zu stellen.

Von Seiten der Union for International Cancer Control (UICC) der WHO erntet man nur Kopfschütteln über das uns

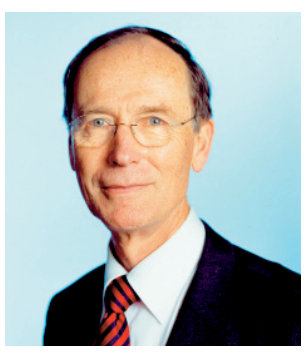

Prof. Dr. med. Ulrich R. Kleeberg

Hämatologisch-onkologische

Praxis Altona, Hamburg

E-Mail: urkleeberg@hopa-hamburg.de

Onkologen beschämende deutsche Unvermögen, das internationale Register endlich einzuführen. Selbst in Entwicklungsländern ist es als entscheidende Maßnahme zur Verbesserung der Gesundheit und Krebsbekämpfung bereits etabliert.

Im September 2011 wurden alle nationalen Regierungen, also auch die von Deutschland, durch die Eingliederung der Krebsbekämpfung in die sog. „Non-communicable Diseases (NCD)“Allianz der WHO zum Aufbau eines KKR verpflichtet.

\section{Gründe für ein Krebsregister}

Es geht darum, Erfolge sowie Fehlentwicklungen aufzudecken, kausale Zusammenhänge für die Entstehung und den Verlauf von Krebs zu erkennen sowie Qualität und Effektivität der primären und sekundären Prävention ebenso wie der diagnostischen und therapeutischen Interventionen zu kontrollieren. Hierfür sind von kritischer Bedeutung:

1. Krebsinzidenzen als Spiegel präventiver Anstrengungen zur Lebensführung und als Richtschnur für eine aktive Gesundheitspolitik,

2. Tumorstadien als Spiegel sekundär präventiver Maßnahmen (Früherkennung),

3. Progressionsfreie Überlebensraten und Folgeerkrankungen als Ausdruck effektiver primärer und adjuvanter Therapien,

4. Remissionsraten und -dauer als Maß für den Nutzen kausaler, tumorgerichteter palliativtherapeutischer Interventionen,

5. Vergleiche zwischen regionalen, nationalen und internationalen onkologischen Institutionen zur Beschreibung der Struktur, Prozess- und Ergebnisqualität der Leistungsträger und nicht zuletzt 


\section{Hier steht eine Anzeige.}

黛 Springer 


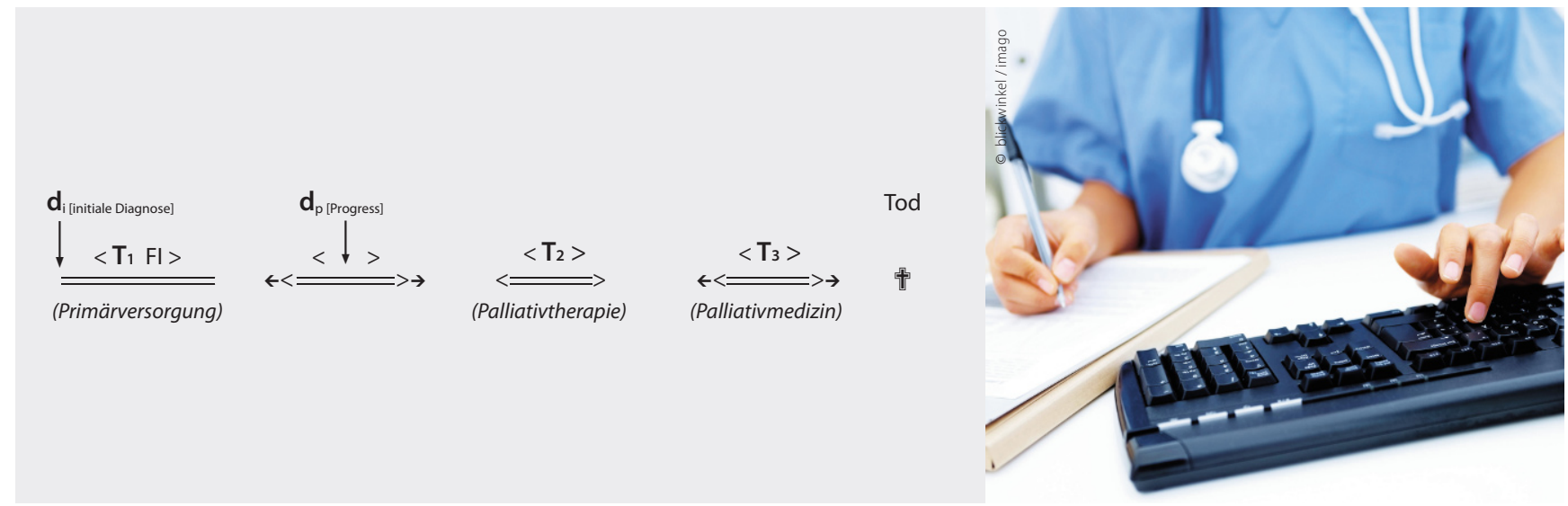

1

Hamburger Vorschlag für einen Stufenplan zum Krebsregister

6. statistische Grundlagen für Kosten- und Wirtschaftlichkeitsanalysen - Voraussetzung für eine rationale, wissenschaftlich legitimierte Priorisierung im Gesundheitswesen.

\section{Stufenplan für sparsame Datenerhebung}

Conditio sine qua non, also unbedingte Voraussetzung für die Akzeptanz der Ärzteschaft ist das „Gebot der Datensparsamkeit“. Ein Stufenplan, wie wir ihn in Hamburg vorschlagen, kann diese unterstützen: Zum Zeitpunkt der Ersterkrankung $\left(\mathrm{d}_{\text {initial }}=\mathrm{d}_{\mathrm{i}}\right.$, Meldung durch Pathologen/Kliniker $)$ und dem Todesdatum kommt zunächst nur das Datum der Aufdeckung eines Progresses beziehungsweise einer Metastasierung $\left(\mathrm{d}_{\text {progress }}=\right.$ $d_{p}$, Rezidiv oder systemische Metastasierung) hinzu (Stufe I). So sind medizinisch und gesundheitspolitisch kritische und für die Qualitätssicherung relevante Schlüsse möglich. Die Zeit $\mathrm{T}_{1}$ von der Erstdiagnose $\left(\mathrm{d}_{\mathrm{i}}\right)$ bis zur Dokumentation eines Progresses $\left(\mathrm{d}_{\mathrm{p}}\right)$ ist der wichtigste Parameter, um Effektivität und Qualität der Primärversorgung zu beschreiben. Die anschließende Phase $\mathrm{T}_{2}$ zwischen dem individuell variablen Zeitpunkt des dokumentierten Progresses $\left(d_{p}\right)$ und dem Tod ist - neben der Lebensqualität - der wesentliche Parameter für die Effektivität und Qualität der tumorgerichteten Palliativtherapie. Diese geht nahtlos in die palliativmedizinische Endphase über $\left(\mathrm{T}_{3}\right)$. Palliativmedizin muss angesichts des begrenzten Nutzens der Palliativtherapie, das Leben zu verlängern, frühzeitig integriert werden $(\triangleright$ Abb. 1).
Auf diese erste folgt Stufe II: Sie besteht nach verbindlicher Regelung der gesetzlichen Finanzierungsgrundlagen darin, das bevölkerungsbezogene KKR unter Anwendung des bereits praktizierten bundesweiten onkologischen Standarddatensatzes (ADT/Gesellschaft der epidemiologischen Krebsregister in Deutschland, GEKID) auszubauen. Wendet man diesen vollständig und flächendeckend an, wird so eine fundierte Bearbeitung spezieller klinisch-wissenschaftlicher und/oder gesundheitspolitischer Fragestellungen möglich. Schrittweise realisieren wir damit gemäß Ziel 8 des NKP eine aussagekräftige Berichterstattung durch ein föderal strukturiertes, nationales klinisches Krebsregister.

So hoffen wir das Beste: Entgegen den Behauptungen ewig Gestriger ist der Nutzen vielfältig wissenschaftlich belegt worden und erlaubt nachhaltige fachliche wie gesundheitspolitische Folgerungen. KKR sind für die Qualitätssicherung unentbehrlich und helfen, „Effektivität“ von „Effizienz“ sowie „Wirkung“ von „Nutzen“ onkologischer Interventionen zu unterscheiden. Diese Differenzierungen sind Voraussetzung für die Weiterentwicklung einer gesundheitsökonomisch zukunftstauglichen optimalen Versorgung Krebskranker.

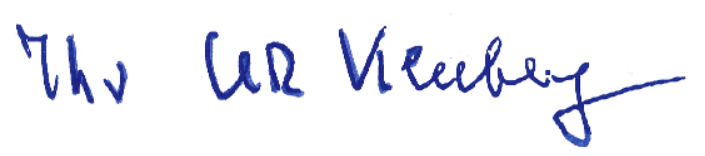

